\title{
Smoothing effect of the homogeneous Boltzmann equation with measure valued initial datum
}

\author{
Yoshinori Morimoto ${ }^{\mathrm{a}, *, 1}$, Tong Yang ${ }^{\mathrm{b}, 2}$ \\ ${ }^{a}$ Graduate School of Human and Environmental Studies, Kyoto University, Kyoto, 606-8501, Japan \\ ${ }^{\mathrm{b}}$ Department of Mathematics, City University of Hong Kong, Hong Kong, PR China
}

Received 29 October 2012; received in revised form 10 November 2013; accepted 18 December 2013

Available online 28 January 2014

\begin{abstract}
We justify the smoothing effect for measure valued solutions to the space homogeneous Boltzmann equation of Maxwellian type cross sections. This is the first rigorous proof of the smoothing effect for any measure valued initial data except the single Dirac mass, which gives the optimal description on the regularity of solutions for positive time, caused by the singularity in the cross section. The main new ingredient in the proof is the introduction of a time degenerate coercivity estimate by using the microlocal analysis.

๑ 2014 L'Association Publications de l'Institut Henri Poincaré. Published by Elsevier B.V. All rights reserved.
\end{abstract}

\section{Résumé}

Nous justifions l'effet régularisant pour les solutions à valeurs mesures de l'équation de Boltzmann spatialement homogène dans le cas des molécules maxwelliennes. Il s'agit de la première preuve rigoureuse de l'effet régularisant pour toutes données initiales à valeurs mesures sauf la masse de Dirac seule, ce qui donne la description optimale de la regularité des solutions en temps positif à causée par la singularité dans le noyau de collision. Le principal ingrédient nouveau dans la preuve est l'introduction d'unc inégalité de coercivité dégénérée par rapport au temps en utilisant l'analyse microlocale.

๑) 2014 L'Association Publications de l'Institut Henri Poincaré. Published by Elsevier B.V. All rights reserved.

MSC: primary 35Q20, 76P05; secondary 35H20, 82B40, 82C40

Keywords: Boltzmann equation; Smoothing effect; Measure initial datum; Coercivity estimate

\section{Introduction}

The purpose of this paper is to analyze the regularizing effect of the Boltzmann equation without angular cutoff in the general setting, that is, for measure valued solutions. Consider the spatially homogeneous Boltzmann equation

\footnotetext{
* Corresponding author. Tel.: +81 75753 6737; fax: +81 757532929.

E-mail addresses: morimoto@math.h.kyoto-u.ac.jp (Y. Morimoto), matyang@cityu.edu.hk (T. Yang).

1 Supported in part by Grant-in-Aid for Scientific Research No. 22540187, Japan Society of the Promotion of Science.

2 Supported in part by the General Research Fund of Hong Kong, CityU No. 103109.
} 


$$
\partial_{t} f(t, v)=Q(f, f)(t, v),
$$

where $f(t, v)$ is the density distribution of particles with velocity $v \in \mathbb{R}^{3}$ at time $t$, and $Q(\cdot, \cdot)$ is the Boltzmann bilinear collision operator given by

$$
Q(g, f)(v)=\iint_{\mathbb{R}^{3}} \int_{\mathbb{S}^{2}} B\left(v-v_{*}, \sigma\right)\left\{g\left(v_{*}^{\prime}\right) f\left(v^{\prime}\right)-g\left(v_{*}\right) f(v)\right\} d \sigma d v_{*},
$$

where the conservation of momentum and energy implies that for $\sigma \in \mathbb{S}^{2}$

$$
v^{\prime}=\frac{v+v_{*}}{2}+\frac{\left|v-v_{*}\right|}{2} \sigma, \quad v_{*}^{\prime}=\frac{v+v_{*}}{2}-\frac{\left|v-v_{*}\right|}{2} \sigma .
$$

In the following, we consider the Cauchy problem of (1.1) with a non-negative initial datum

$$
f(0, v)=f_{0}(v) .
$$

Here, $f_{0}(v)$ is a density of probability distribution (more generally a probability measure).

The non-negative cross section $B(z, \sigma)$ in the collision operator depends only on $|z|$ and the scalar product $\frac{z}{|z|} \cdot \sigma$. Motivated by the physical model of potential of inverse power laws, we assume

$$
B\left(\left|v-v_{*}\right|, \cos \theta\right)=\Phi\left(\left|v-v_{*}\right|\right) b(\cos \theta), \quad \cos \theta=\frac{v-v_{*}}{\left|v-v_{*}\right|} \cdot \sigma, 0 \leqslant \theta \leqslant \frac{\pi}{2},
$$

where

$$
\begin{aligned}
& \Phi(|z|)=\Phi_{\gamma}(|z|)=|z|^{\gamma}, \quad \text { for some } \gamma>-3, \\
& b(\cos \theta) \theta^{2+2 s} \rightarrow K \quad \text { when } \theta \rightarrow 0+, \text { for } 0<s<1 \text { and } K>0 .
\end{aligned}
$$

In fact, if the inter-particle potential $U(\rho)$ is proportional to $\rho^{-(q-1)}$ with $q>2$, where $\rho$ denotes the distance between two interacting particles, then $s$ and $\gamma$ are given by

$$
s=1 /(q-1)<1, \quad \gamma=1-4 s=1-4 /(q-1)>-3 .
$$

For this physical model, we have $\gamma=0$ and $s=1 / 4$ when $q=5$, which is called the Maxwellian molecule. Inspired by this case, in this paper, we consider the Maxwellian molecule type cross section when

$$
\gamma=0, \quad 0<s<1 \text {. }
$$

The angle $\theta$ in the cross section is the deviation angle, i.e., the angle between pre- and post-collisional velocities. Even though the range of $\theta$ is in an interval $[0, \pi]$, as in [21], it is customary to restrict it to $[0, \pi / 2]$, by replacing $b(\cos \theta)$ by its "symmetrized" version

$$
[b(\cos \theta)+b(\cos (\pi-\theta))] \mathbf{1}_{0 \leqslant \theta \leqslant \pi / 2}
$$

because of the invariance of the product $f\left(v^{\prime}\right) f\left(v_{*}^{\prime}\right)$ in the collision operator $Q(f, f)$ under the change of variables $\sigma \rightarrow-\sigma$.

One of the important feature of the cross section without angular cutoff is that $b(\cos \theta)$ has the integrable singularity, that is,

$$
\int_{\mathbb{S}^{2}} b\left(\frac{v-v_{*}}{\left|v-v_{*}\right|} \cdot \sigma\right) d \sigma=2 \pi \int_{0}^{\pi / 2} b(\cos \theta) \sin \theta d \theta=\infty .
$$

This kind of singularity leads to some difficulties in the study of the existence and solution behavior because the gain and loss terms in the collision operator cannot be considered separately. Moreover, the angular singularity also leads to the gain of regularity in the solution. For later analysis, as before, the case where $0<s<1 / 2$, that is, $\int_{0}^{\pi / 2} \theta b(\cos \theta) \sin \theta d \theta<\infty$ is called the mild singularity, and another case $1 / 2 \leqslant s<1$ is called the strong singularity. Note that to handle the strong singularity, some symmetry property of the collision operator should be used. 
The study on the homogeneous Boltzmann equation has a very long history, cf. [7,5] and the references in recent work [12]. In particular, the smoothing effect of (weak) solutions to the Cauchy problem for the non cutoff homogeneous Boltzmann equation has been studied by many authors in $[9,2,3,15,10,4,8]$, including Gevrey smoothing effect in [16]. However, the problem for measure initial data has been studied only in [14], when it consists of a sum of four Dirac masses.

On the other hand, in [22] Villani conjectured that the regularizing effect for weak measurable solutions holds for any measure initial data except a single Dirac mass. This is a much stronger statement than the previous works on the weighted $L^{p}$ solutions because one has to consider measure valued solutions. The purpose of this paper is to justify this conjecture, which is optimal in the sense that a single Dirac mass is a stationary solution of the Boltzmann equation.

Let us now introduce some notations for function spaces and recall some related works on the existence and uniqueness. For every $0 \leqslant \alpha<\infty$, we denote by $P_{\alpha}\left(\mathbb{R}^{d}\right)$ the class of all probability measure $F$ on $\mathbb{R}^{d}, d \geqslant 1$, such that

$$
\int_{\mathbb{R}^{d}}|v|^{\alpha} d F(v)<\infty .
$$

Concerning the Cauchy problem for the homogeneous Boltzmann equation of the Maxwellian molecule type cross section, Tanaka [18] in 1978 proved the existence and the uniqueness of the solution in the space $P_{2}\left(\mathbb{R}^{d}\right)$ by using probability theory. The proof of this result was simplified and generalized in $[17,19]$.

The existence of solution with bounded energy was extended in [6] to the initial datum as a probability measure with infinite energy. Precisely, following [6], introduce

Definition 1.1. A function $\psi: \mathbb{R}^{3} \rightarrow \mathbb{C}$ is called a characteristic function if there is a probability measure $\Psi$ (i.e., a positive Borel measure with $\left.\int_{\mathbb{R}^{3}} d \Psi(v)=1\right)$ such that the identity $\psi(\xi)=\int_{\mathbb{R}^{3}} e^{-i v \cdot \xi} d \Psi(v)$ holds. We denote the set of all characteristic functions by $\mathcal{K}$.

Following [19], a subspace $\mathcal{K}^{\alpha}$ for $\alpha \geqslant 0$ was defined in [6] as follows:

$$
\mathcal{K}^{\alpha}=\left\{\varphi \in \mathcal{K} ;\|\varphi-1\|_{\alpha}<\infty\right\}
$$

where

$$
\|\varphi-1\|_{\alpha}=\sup _{\xi \in \mathbb{R}^{3}} \frac{|\varphi(\xi)-1|}{|\xi|^{\alpha}} .
$$

The space $\mathcal{K}^{\alpha}$ endowed with the distance

$$
\|\varphi-\tilde{\varphi}\|_{\alpha}=\sup _{\xi \in \mathbb{R}^{3}} \frac{|\varphi(\xi)-\tilde{\varphi}(\xi)|}{|\xi|^{\alpha}}
$$

is a complete metric space (see Proposition 3.10 of [6]). It follows that $\mathcal{K}^{\alpha}=\{1\}$ for all $\alpha>2$ and the embeddings (Lemma 3.12 of [6]) hold, that is,

$$
\{1\} \subset \mathcal{K}^{\alpha} \subset \mathcal{K}^{\beta} \subset \mathcal{K}^{0}=\mathcal{K} \text { for all } 2 \geqslant \alpha \geqslant \beta \geqslant 0 .
$$

The definition of the space $\mathcal{K}^{\alpha}$ is natural because we have the following lemma (Lemma 3.15 of [6]).

Lemma 1.2. Let $\Psi$ be a probability measure on $\mathbb{R}^{3}$ such that

$$
\exists \alpha \in(0,2] ; \quad \int|v|^{\alpha} d \Psi(v)<\infty, \quad \text { and moreover, } \quad \int v_{j} d \Psi(v)=0, \quad j=1,2,3, \text { when } \alpha>1 .
$$

Then the Fourier transform of $\Psi$, that is, $\psi(\xi)=\int e^{-i v \cdot \xi} d \Psi(v)$ belongs to $\mathcal{K}^{\alpha}$.

The inverse of the lemma does not hold, in fact, the space $\mathcal{K}^{\alpha}$ is bigger than the set of the Fourier transform of $P_{\alpha}$ (Remark 3.16 of [6]). So we introduce $\tilde{P}_{\alpha}=\mathcal{F}^{-1}\left(\mathcal{K}^{\alpha}\right)$ endowed also with the distance (1.7). The existence and the 
uniqueness of the solution in the space $\tilde{P}_{\alpha}$ was proved in [6] for the mild singularity, and has been recently improved in [14] for the strong singularity. Namely, if the cross section $b(\cos \theta)$ satisfies (1.3) with $0<s<1$ and if $2 s<\alpha \leqslant 2$, then there exists a unique solution to the Cauchy problem $(1.1)-(1.2)$ in the space $C\left([0, \infty), \tilde{P}_{\alpha}\right)$ for any initial datum in $\tilde{P}_{\alpha}$ (see Theorem A.1 in Appendix A).

We are now ready to state the main results of this paper.

Theorem 1.3. Let $b(\cos \theta)$ satisfy (1.3) with $0<s<1$ and let $\alpha \in(2 s, 2]$. If $F_{0} \in \tilde{P}_{\alpha}\left(\mathbb{R}^{3}\right)$ is not a single Dirac mass and $f(t, v)$ is a unique solution in $C\left([0, \infty), \tilde{P}_{\alpha}\right)$ to the Cauchy problem $(1.1)-(1.2)$, then there exists a $T>0$ such that $f(t, \cdot) \in H^{\infty}\left(\mathbb{R}^{3}\right)$ for any $0<t \leqslant T$. Moreover, if $F_{0} \in P_{2}\left(\mathbb{R}^{3}\right)$ then $T=\infty$.

Lemma 1.4. Let $F_{0} \in \tilde{P}_{\alpha}\left(\mathbb{R}^{3}\right)$ and $f(t, v) \in C\left([0, \infty), \tilde{P}_{\alpha}\right)$ be the same as in Theorem 1.3. If $\psi(t, \xi)$ and $\psi_{0}(\xi)$ are Fourier transforms of $f(t, v)$ and $F_{0}$, respectively, then there exist $T>0$ and $C>0$, such that for $t \in[0, T]$ we have

$$
t \int_{\mathbb{R}^{3}}\langle\xi\rangle^{2 s}|h(\xi)|^{2} d \xi \leqslant C\left(\int_{\mathbb{R}^{3}}\left(\int_{\mathbb{S}^{2}} b\left(\frac{\xi}{|\xi|} \cdot \sigma\right)\left(1-\left|\psi\left(t, \xi^{-}\right)\right|\right) d \sigma\right)|h(\xi)|^{2} d \xi+\int_{\mathbb{R}^{3}}|h(\xi)|^{2} d \xi\right), \quad \text { for } \forall h \in L_{s}^{2},
$$

where $\xi^{-}=(\xi-|\xi| \sigma) / 2$.

With Lemma 1.4, the proof of Theorem 1.3 can be given as follows.

Proof of Theorem 1.3. It follows from the Bobylev formula that the Cauchy problem (1.1)-(1.2) is reduced to

$$
\left\{\begin{array}{l}
\partial_{t} \psi(t, \xi)=\int_{\mathbb{S}^{2}} b\left(\frac{\xi \cdot \sigma}{|\xi|}\right)\left(\psi\left(t, \xi^{+}\right) \psi\left(t, \xi^{-}\right)-\psi(t, \xi) \psi(t, 0)\right) d \sigma \\
\psi(0, \xi)=\psi_{0}(\xi), \quad \text { where } \xi^{ \pm}=\frac{\xi}{2} \pm \frac{|\xi|}{2} \sigma
\end{array}\right.
$$

By Theorem A.1, $\psi(t, \xi) \in C\left([0, \infty), \mathcal{K}^{\alpha}\right)$. Define a time dependent weight function

$$
M_{\delta}(t, \xi)=\langle\xi\rangle^{N t^{2}-4}\langle\delta \xi\rangle^{-2 N_{0}}, \quad\langle\xi\rangle^{2}=1+|\xi|^{2},
$$

where $N_{0}=N T^{2} / 2+2, N \in \mathbb{N}$ and $\delta>0$. We multiply the first equation of (1.10) by $M_{\delta}(t, \xi)^{2} \overline{\psi(t, \xi)}$ and integrate with respect to $\xi$ over $\mathbb{R}^{3}$. Denote $\psi^{ \pm}=\psi\left(t, \xi^{ \pm}\right)$and $M^{+}=M_{\delta}\left(t, \xi^{+}\right)$to simplify the notation and note that

$$
\begin{aligned}
-2 \operatorname{Re}\left\{\left(\psi^{+} \psi^{-}-\psi\right) M^{2} \bar{\psi}\right\}= & \left(|M \psi|^{2}+\left|M^{+} \psi^{+}\right|^{2}-2 \operatorname{Re}\left\{\psi^{-}\left(M^{+} \psi^{+}\right) \overline{M \psi}\right\}\right) \\
& +\left(|M \psi|^{2}-\left|M^{+} \psi^{+}\right|^{2}\right)+2 \operatorname{Re}\left\{\psi^{-}\left(\left(M-M^{+}\right) \psi^{+}\right) \overline{M \psi}\right\} \\
= & J_{1}+J_{2}+J_{3} .
\end{aligned}
$$

Using the Cauchy-Schwarz inequality for the third term of $J_{1}$, we have

$$
J_{1} \geqslant\left(1-\left|\psi^{-}\right|\right)\left(|M \psi|^{2}+\left|M^{+} \psi^{+}\right|^{2}\right) \geqslant\left(1-\left|\psi^{-}\right|\right)|M \psi|^{2} .
$$

Therefore, by means of (1.9) we get

$$
\int_{\mathbb{R}^{3} \times \mathbb{S}^{2}} b\left(\frac{\xi}{|\xi|} \cdot \sigma\right) J_{1} d \sigma d \xi+\int_{\mathbb{R}^{3}}|M \psi|^{2} d \xi \gtrsim t \int_{\mathbb{R}^{3}}\langle\xi\rangle^{2 s}|M \psi|^{2} d \xi
$$

where $A \gtrsim B$ means that there exists a constant $C_{0}>0$ such that $A \geqslant C_{0} B$. If we use the change of variable $\xi \rightarrow \xi^{+}$ for the term $M^{+} \psi^{+}$in $J_{2}$, by the cancellation lemma (Lemma 1 of [1]), we have

$$
\left|\int_{\mathbb{R}^{3} \times \mathbb{S}^{2}} b\left(\frac{\xi}{|\xi|} \cdot \sigma\right) J_{2} d \sigma d \xi\right|=\left.\left.2 \pi\left|\int_{\mathbb{R}^{3}}\right| M \psi\right|^{2}\left(\int_{0}^{\pi / 2} b(\cos \theta) \sin \theta\left(1-\frac{1}{\cos ^{3}(\theta / 2)}\right) d \theta\right) d \xi\left|\lesssim \int_{\mathbb{R}^{3}}\right| M \psi\right|^{2} d \xi,
$$


where $A \lesssim B$ means that there exists a constant $C_{0}>0$ such that $A \leqslant C_{0} B$. Since $\left|M-M^{+}\right| \lesssim \sin ^{2}(\theta / 2) M^{+}$(see (3.4) of [15]), by the Cauchy-Schwarz inequality we also have the same upper bound estimate for $J_{3}$ by using again the change of variable $\xi \rightarrow \xi^{+}$for the term including $M^{+} \psi^{+}$. Since

$$
2 \operatorname{Re}\left(\frac{\partial \psi}{\partial t} M^{2} \bar{\psi}\right)=\frac{\partial|M \psi|^{2}}{\partial t}-4 N t \log \langle\xi\rangle|M \psi|^{2},
$$

and $|\xi|^{2 s} / \log \langle\xi\rangle \rightarrow \infty$ as $|\xi| \rightarrow \infty$, we have

$$
\frac{d}{d t} \int_{\mathbb{R}^{3}}\left|M_{\delta}(t, \xi) \psi(t, \xi)\right|^{2} d \xi \lesssim \int_{\mathbb{R}^{3}}\left|M_{\delta}(t, \xi) \psi(t, \xi)\right|^{2} d \xi
$$

which gives for $t \in(0, T]$

$$
\int_{\mathbb{R}^{3}}\left|\langle\xi\rangle^{N t^{2}-4}\left(1+\delta|\xi|^{2}\right)^{-N_{0}} \psi(t, \xi)\right|^{2} d \xi \lesssim \int_{\mathbb{R}^{3}}\left|\langle\xi\rangle^{-4} \psi_{0}(\xi)\right|^{2} d \xi
$$

Letting $\delta \rightarrow 0$, we obtain the first part of Theorem 1.3 because we can take an arbitrarily large $N$.

We now turn to the second part of the theorem when $F_{0} \in P_{2}\left(\mathbb{R}^{3}\right)$. We notice that the energy of solution is uniformly bounded by that of the initial datum (see Proposition A.2 in Appendix A), so that we have $\int|v|^{2} f(T, v) d v \leqslant$ $\int|v|^{2} d F_{0}(v)$ for a $T>0$ given in Lemma 1.4. In view of $f(T, v) \in L^{\infty}\left(\mathbb{R}^{3}\right)$ we obtain

$$
\|f(T)\|_{L \log L}:=\int f(T, v) \log (1+f(T, v)) d v<\infty
$$

so that $f(T) \in L_{2}^{1} \cap L \log L$. It follows from Theorem 1 in [20] that

$$
\sup _{t \geqslant T}\left(\|f(t)\|_{L_{2}^{1}}+\|f(t)\|_{L \log L}\right)<\infty,
$$

which shows that there exists a $\kappa>0$ independent of $t \geqslant T$ such that

$$
1-|\psi(t, \xi)| \geqslant \kappa \min \left(1,|\xi|^{2}\right)
$$

by means of Lemma 3 in [1]. Therefore, for $|\xi| \geqslant R$ for some $R>0$ suitably large, we have

$$
\int_{\mathbb{S}^{2}} b\left(\frac{\xi}{|\xi|} \cdot \sigma\right)\left(1-\left|\psi\left(t, \xi^{-}\right)\right|\right) d \sigma \geqslant 2 \pi \kappa \int_{0}^{|\xi|^{-1}} b(\cos \theta)\left|\xi^{-}\right|^{2} \sin \theta d \theta \gtrsim|\xi|^{2} \int_{0}^{|\xi|^{-1}} \theta^{1-2 s} d \theta \gtrsim|\xi|^{2 s}
$$

which gives the standard coercivity estimate instead of (1.9). Hence this leads us to $f(t, v) \in H^{\infty}\left(\mathbb{R}^{3}\right)$ for $\forall t>T$ by the same argument used in [15].

The rest of the paper will be organized as follows. In the next section, we will prove Lemma 1.4 about the degenerate coercivity estimate which is the key estimate to show the smoothing effect. And in Appendix A, we will recall the existence and uniqueness result obtained in $[6,14]$ and show the continuity of the time derivative of the solution which is needed in Section 2. It will be also shown in Appendix A that the energy of the solution for the initial datum $F_{0} \in P_{2}\left(\mathbb{R}^{3}\right)$ is bounded.

\section{Degenerate coercivity estimate}

To obtain the coercivity estimate for measure valued function which is not concentrated at a single point, we will consider two cases, that is, the case when the measure is concentrated on a straight line and otherwise. Unlike the standard coercivity estimate obtained in the previous works, the key observation is that the coercivity estimate is degenerate in the time variable as shown in Lemma 1.4. That is, one cannot expect to have a gain of regularity of order $2 s$ uniformly up to initial time. For this, we need to consider the time derivative of $\psi\left(t, \xi^{-}\right)$in the case when $\xi$ is parallel to the straight line of the concentration of the measure. For clear presentation, the coercivity is estimated in the following two subsections. 


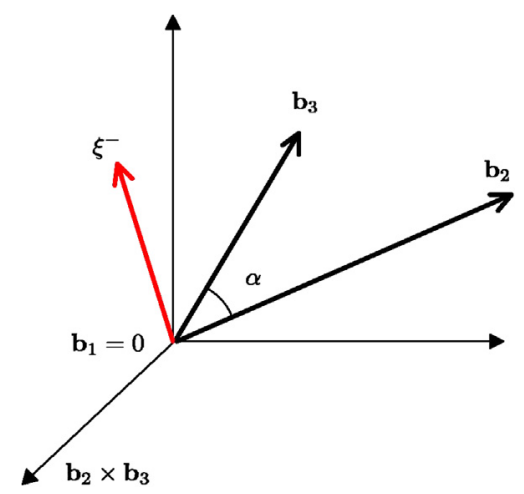

Fig. 1. $\xi^{-}$and three vectors $\mathbf{b}_{1}, \mathbf{b}_{2}, \mathbf{b}_{3}$.

\subsection{Initial measure not concentrated on a straight line}

We now consider the case when $F_{0}(v)$ is not concentrated on a straight line. In this case, without loss of generality, we can assume that there exist three small balls denoted by $A_{i}=B\left(\mathbf{b}_{i}, \delta\right)$ with center at $v=\mathbf{b}_{i}$ and radius $\delta>0$ such that $\int_{A_{i}} d F_{0}(v)=m_{i}>0$, for $i=1,2,3$. Up to a linear coordinate transform, we can assume $\mathbf{b}_{1}=\mathbf{0}, \mathbf{b}_{2}$ and $\mathbf{b}_{3}$ are linearly independent. That is

$$
\eta_{0}=1-\left|\frac{\mathbf{b}_{2}}{\left|\mathbf{b}_{2}\right|} \cdot \frac{\mathbf{b}_{3}}{\left|\mathbf{b}_{3}\right|}\right|=1-|\cos \alpha|>0
$$

where $\alpha$ is the angle between $\mathbf{b}_{2}$ and $\mathbf{b}_{3}$ (see Fig. 1). Take two positive constants $d_{1}<d_{2}$ such that

$$
0<d_{1} \min \left\{\left|\mathbf{b}_{2}\right|,\left|\mathbf{b}_{3}\right|\right\}<d_{2} \max \left\{\left|\mathbf{b}_{2}\right|,\left|\mathbf{b}_{3}\right|\right\} \leqslant \frac{\pi}{2} .
$$

Put $d=\left(d_{1}+d_{2}\right) / 2$. Firstly, we assume that $\xi^{-}$varies on the circle

$$
\mathcal{C}=\left\{\xi \in \mathbb{R}^{3} ;|\xi|=d, \quad \xi \perp\left(\mathbf{b}_{2} \times \mathbf{b}_{3}\right)\right\}
$$

In the following discussion, we choose $\delta>0$ to be sufficiently small.

Denote

$$
\int_{A_{j}} e^{-i v \cdot \xi^{-}} d F(v)=m_{j}\left(a_{j}+i b_{j}\right), \quad j=1,2,3 .
$$

Note that $\left|a_{j}+i b_{j}\right| \leqslant 1$. With the above notations, it is straightforward to check that

$$
\begin{aligned}
& \left(a_{1}, b_{1}\right)=(1,0)+\mathbf{e}_{1}, \\
& \left(a_{2}, b_{2}\right)=\left(\cos \left(\left|\xi^{-}\right|\left|\mathbf{b}_{2}\right| \cos \gamma_{1}\right), \sin \left(\left|\xi^{-}\right|\left|\mathbf{b}_{2}\right| \cos \gamma_{1}\right)\right)+\mathbf{e}_{2}, \\
& \left(a_{3}, b_{3}\right)=\left(\cos \left(\left|\xi^{-}\right|\left|\mathbf{b}_{3}\right| \cos \gamma_{2}\right), \sin \left(\left|\xi^{-}\right|\left|\mathbf{b}_{3}\right| \cos \gamma_{2}\right)\right)+\mathbf{e}_{3},
\end{aligned}
$$

where $\gamma_{1}$ is the angle between the vectors $\xi^{-}$and $\mathbf{b}_{2}, \gamma_{2}$ is the angle between the vectors $\xi^{-}$and $\mathbf{b}_{3},\left|\mathbf{e}_{i}\right|=0(1) \delta$, $i=1,2,3$. Notice that $\gamma_{2}=\gamma_{1} \pm \alpha$. With the above choice of parameters, we have when $\delta$ is sufficiently small,

$$
\begin{aligned}
2 & -\left|\frac{\left(a_{1}, b_{1}\right)}{\left|\left(a_{1}, b_{1}\right)\right|} \cdot \frac{\left(a_{2}, b_{2}\right)}{\left|\left(a_{2}, b_{2}\right)\right|}\right|-\left|\frac{\left(a_{1}, b_{1}\right)}{\left|\left(a_{1}, b_{1}\right)\right|} \cdot \frac{\left(a_{3}, b_{3}\right)}{\left|\left(a_{3}, b_{3}\right)\right|}\right| \\
& =2-\cos \left(\left|\xi^{-}\right|\left|\mathbf{b}_{2}\right| \cos \gamma_{1}\right)-\cos \left(\left|\xi^{-}\right|\left|\mathbf{b}_{3}\right| \cos \left(\gamma_{1} \pm \alpha\right)\right)+0(1) \delta \geqslant c_{0} \eta_{0},
\end{aligned}
$$

where $c_{0}>0$ is a constant independent of $\delta$. Hence, if $\psi_{0}(\xi)=\int e^{-i v \cdot \xi} d F_{0}(v)$ and $\xi^{-}$varies on $\mathcal{C}$ defined by (2.1), then we have 


$$
\begin{aligned}
\psi_{0}(0)-\left|\psi_{0}\left(\xi^{-}\right)\right| & =1-\left|\int_{A^{c} \bigcup_{j=1}^{3} A_{j}} e^{-i v \cdot \xi^{-}} d F_{0}(v)\right| \\
& \geqslant \sum_{j=1}^{3} \int_{A_{j}} d F_{0}(v)-\left|\sum_{j=1}^{3} \int_{A_{j}} e^{-i v \cdot \xi^{-}} d F_{0}(v)\right| \\
& =\sum_{j=1}^{3} m_{j}-\left|\sum_{j=1}^{3} m_{j}\left(a_{j}+i b_{j}\right)\right| \\
& \geqslant \min \left\{m_{1}, m_{2}, m_{3}\right\}\left(3-\left|\sum_{j=1}^{3}\left(a_{j}+i b_{j}\right)\right|\right) \\
& \geqslant \frac{1}{3} \min \left\{m_{1}, m_{2}, m_{3}\right\}\left\{2-\left|\frac{\left(a_{1}, b_{1}\right)}{\left|\left(a_{1}, b_{1}\right)\right|} \cdot \frac{\left(a_{2}, b_{2}\right)}{\left|\left(a_{2}, b_{2}\right)\right|}\right|-\left|\frac{\left(a_{1}, b_{1}\right)}{\left|\left(a_{1}, b_{1}\right)\right|} \cdot \frac{\left(a_{3}, b_{3}\right)}{\left|\left(a_{3}, b_{3}\right)\right|}\right|\right\} \\
& \geqslant \frac{1}{3} \min \left\{m_{1}, m_{2}, m_{3}\right\} c_{0} \eta_{0}:=\kappa_{0},
\end{aligned}
$$

because $\left|a_{j}+i b_{j}\right| \leqslant 1$ and

$$
\begin{aligned}
\left|\sum_{j=1}^{3}\left(a_{j}+i b_{j}\right)\right|^{2} \leqslant & \left(\left|a_{1}+i b_{1}\right|+\sum_{j=2}^{3}\left|a_{j}+i b_{j}\right|\left|\frac{\left(a_{1}, b_{1}\right)}{\left|\left(a_{1}, b_{1}\right)\right|} \cdot \frac{\left(a_{j}, b_{j}\right)}{\left|\left(a_{j}, b_{j}\right)\right|}\right|\right)^{2} \\
& +\left(\sum_{j=2}^{3}\left|a_{j}+i b_{j}\right|\left|\frac{\left(a_{1}, b_{1}\right)}{\left|\left(a_{1}, b_{1}\right)\right|} \times \frac{\left(a_{j}, b_{j}\right)}{\left|\left(a_{j}, b_{j}\right)\right|}\right|\right)^{2} \\
\leqslant & \left(1+\sum_{j=2}^{3}\left|\frac{\left(a_{1}, b_{1}\right)}{\left|\left(a_{1}, b_{1}\right)\right|} \cdot \frac{\left(a_{j}, b_{j}\right)}{\left|\left(a_{j}, b_{j}\right)\right|}\right|\right)^{2}+\left(\sum_{j=2}^{3}\left|\frac{\left(a_{1}, b_{1}\right)}{\left|\left(a_{1}, b_{1}\right)\right|} \times \frac{\left(a_{j}, b_{j}\right)}{\left|\left(a_{j}, b_{j}\right)\right|}\right|\right)^{2} \\
\leqslant & 5+2 \sum_{j=2}^{3}\left|\frac{\left(a_{1}, b_{1}\right)}{\left|\left(a_{1}, b_{1}\right)\right|} \cdot \frac{\left(a_{j}, b_{j}\right)}{\left|\left(a_{j}, b_{j}\right)\right|}\right| .
\end{aligned}
$$

Since $\psi(t, \xi)$ is continuous (see Theorem A.1 in Appendix A) and $\psi(0, \xi)=\psi_{0}(\xi)$, by means of (2.2), there exist $\mu>0, \varepsilon>0$ and $T>0$ such that for any $\xi^{-}$belonging to the set

$$
\mathcal{C}_{\mu, \varepsilon}=\left\{\eta \in \mathbb{R}^{3} ; d-\mu \leqslant|\eta| \leqslant d+\mu,\left|\frac{\eta}{|\eta|} \cdot\left(\frac{\mathbf{b}_{2} \times \mathbf{b}_{3}}{\left|\mathbf{b}_{2} \times \mathbf{b}_{3}\right|}\right)\right| \leqslant \varepsilon\right\},
$$

we have

$$
1-\left|\psi\left(t, \xi^{-}\right)\right| \geqslant \kappa_{0} / 2 \quad \text { for } t \in[0, T] .
$$

Take a $R>0$ such that $(d+\mu) / R=\varepsilon / 10$. Let $|\xi| \geqslant R$, and for $\omega=\xi /|\xi| \in \mathbb{S}^{2}$ take the coordinate $\sigma=(\theta, \phi) \in$ $[0, \pi / 2] \times[0,2 \pi]$ with the pole $\omega$. Write

$$
\xi^{-}=\frac{\xi}{2}-\frac{|\xi|}{2} \sigma=\xi^{-}(\theta, \phi) .
$$

If $\theta$ satisfies

$$
d-\mu \leqslant\left|\xi^{-}(\theta, \phi)\right|=|\xi| \sin \frac{\theta}{2} \leqslant d+\mu
$$

then there exists an interval $I_{\omega} \subset[0,2 \pi]$ such that $\xi^{-}(\theta, \phi) \in \mathcal{C}_{\mu, \varepsilon}$ for $\phi \in I_{\omega}$ because $\theta / 2 \leqslant \sin ^{-1}(d+\mu) / R<\varepsilon / 5$ and the set

$$
\left\{\lambda \xi^{-}(\theta, \phi) \in \mathbb{R}^{3} ; \phi \in[0,2 \pi], 0 \leqslant \lambda \leqslant 1\right\}
$$

intersects the plane spanned by $\mathbf{b}_{2}$ and $\mathbf{b}_{3}$ when $\left|\omega \cdot\left(\mathbf{b}_{2} \times \mathbf{b}_{3}\right) /\right| \mathbf{b}_{2} \times \mathbf{b}_{3}||<\cos \theta / 2$ (see Fig. 2). 


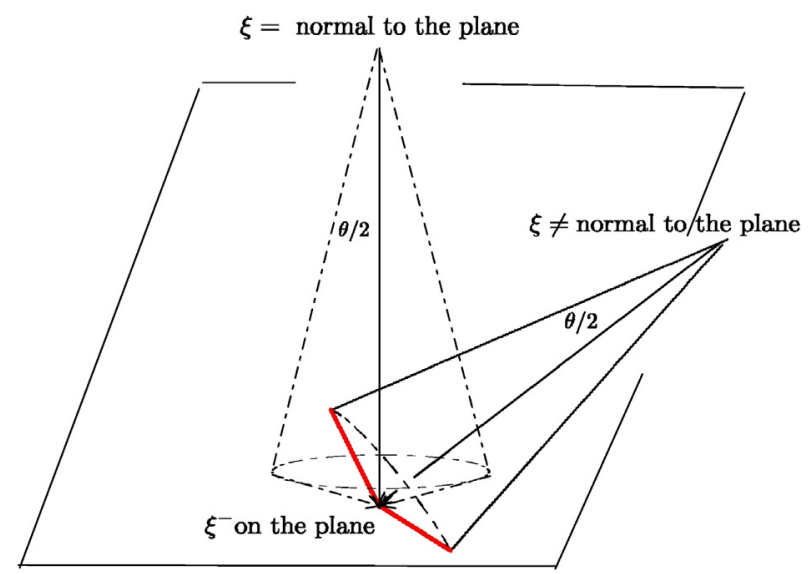

Fig. 2. Intersection between $\left\{\xi^{-}\right\}$and the plane spanned by $\mathbf{b}_{2}, \mathbf{b}_{3}$.

It is obvious that the interval $I_{\omega}$ plays the same role for $\tilde{\omega} \in \mathbb{S}^{2}$ close to $\omega$. Therefore, for any $\xi$ belonging to a conic neighborhood of $\omega$

$$
\Gamma_{\omega}=\left\{\xi \in \mathbb{R}^{3} ;\left|\frac{\xi}{|\xi|}-\omega\right|<\varepsilon_{\omega},|\xi| \geqslant R\right\}
$$

with a sufficiently small $\varepsilon_{\omega}>0$, we have

$$
\begin{aligned}
\int_{\mathbb{R}^{3}}\left(\int_{\mathbb{S}^{2}} b\left(\frac{\xi}{|\xi|} \cdot \sigma\right)\left(1-\mid \psi\left(t, \xi^{-} \mid\right)\right) d \sigma\right)|h(\xi)|^{2} d \xi & \gtrsim \int_{\Gamma_{\omega}}\left(\int_{I_{\omega}} d \phi \int_{2 \sin ^{-1}(d-\mu) /|\xi|}^{2 \sin ^{-1}(d+\mu) /|\xi|} \theta^{-1-2 s} \frac{\kappa_{0}}{2} d \theta\right)|h(\xi)|^{2} d \xi \\
& \gtrsim \int_{\Gamma_{\omega}}|\xi|^{2 s}|h(\xi)|^{2} d \xi
\end{aligned}
$$

which together with the standard covering argument on $\mathbb{S}^{2}$ yields

$$
\int_{\mathbb{R}^{3}}\left(\int_{\mathbb{S}^{2}} b\left(\frac{\xi}{|\xi|} \cdot \sigma\right)\left(1-\mid \psi\left(t, \xi^{-} \mid\right)\right) d \sigma\right)|h(\xi)|^{2} d \xi+\int_{\mathbb{R}^{3}}|h(\xi)|^{2} d \xi \gtrsim \int_{\mathbb{R}^{3}}\langle\xi\rangle^{2 s}|h(\xi)|^{2} d \xi,
$$

if $t \in[0, T]$.

\subsection{Initial measure concentrated on a straight line}

We now consider the case when $F_{0}(v)$ is concentrated on a straight line and not equal to a single Dirac measure. By means of a suitable choice of the coordinate we may assume that $F_{0}(v)=\delta\left(v^{\prime}\right) F_{03}\left(v_{3}\right)$ and its Fourier transform $\psi_{0}(\xi)=\psi_{03}\left(\xi_{3}\right)$, where $\psi_{03}$ is the Fourier transform of $F_{03}$. Since $F_{03}\left(v_{3}\right)$ is not a point Dirac measure in $\mathbb{R}$, it follows from Corollary 3.5.11 in [11] that there exists a $\xi_{03}>0$ such that $\left|\psi_{03}\left( \pm \xi_{03}\right)\right|<1$, in view of $\psi(-\xi)=\overline{\psi(\xi)}$. By means of the continuity of $\psi$, there exist $0<\kappa<1$ and $0<a_{1}<a_{2}$ such that

$$
\left|\psi_{0}\left(\xi^{\prime}, \xi_{3}\right)\right| \leqslant 1-\kappa, \quad \text { for } \forall \xi^{\prime} \in \mathbb{R}^{2}, \forall \xi_{3} \in \mathbb{R} \text { with } a_{1} \leqslant\left|\xi_{3}\right| \leqslant a_{2} .
$$

We now split the discussion into two cases.

\subsubsection{The case when $\xi^{-}$is almost orthogonal to the third axis}

For the sake of simplicity, we denote $\xi^{-}$by $\xi$ throughout this subsection except for the case when confusion might occur. We also denote $\psi$ instead of $\psi_{0}$ for brevity. 


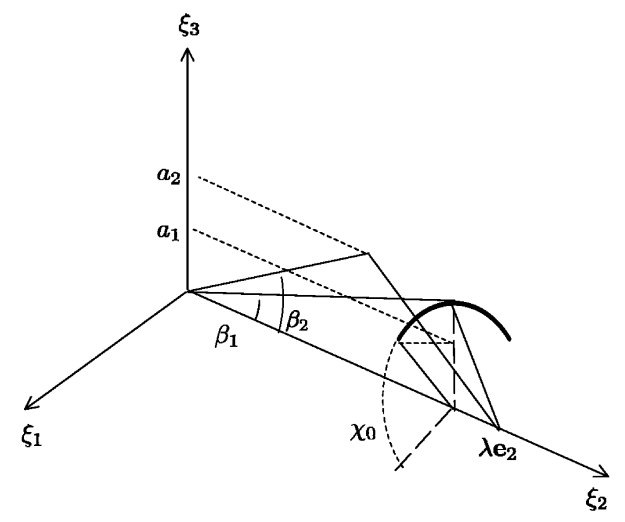

Fig. 3. $\xi^{-}$with $\beta=\beta_{1}$ and $\chi \in\left[\chi_{0}, \pi-\chi_{0}\right]$.

Note that

$$
\begin{aligned}
\left(\partial_{t}|\psi|^{2}\right)(0, \xi) & =2 \operatorname{Re} \int_{\mathbb{S}^{2}} b\left(\frac{\xi}{|\xi|} \cdot \sigma\right)\left(\psi^{+} \psi^{-} \bar{\psi}-|\psi|^{2}\right) d \sigma \\
& =-\int_{\mathbb{S}^{2}} b\left(\frac{\xi}{|\xi|} \cdot \sigma\right)\left(\left|\psi^{+}\right|^{2}+|\psi|^{2}-2 \operatorname{Re}\left\{\psi^{-} \psi^{+} \bar{\psi}\right\}\right) d \sigma+\int_{\mathbb{S}^{2}} b\left(\frac{\xi}{|\xi|} \cdot \sigma\right)\left(\left|\psi^{+}\right|^{2}-|\psi|^{2}\right) d \sigma \\
& \leqslant-\int_{\mathbb{S}^{2}} b\left(\frac{\xi}{|\xi|} \cdot \sigma\right)\left(1-\left|\psi^{-}\right|\right)\left(\left|\psi^{+}\right|^{2}+|\psi|^{2}\right) d \sigma+\int_{\mathbb{S}^{2}} b\left(\frac{\xi}{|\xi|} \cdot \sigma\right)\left(\left|\psi^{+}\right|^{2}-|\psi|^{2}\right) d \sigma .
\end{aligned}
$$

If we put $\xi=\lambda \mathbf{e}_{2}(\lambda>0)$ in the above estimate and take the polar coordinate $\sigma=(2 \beta, \chi) \in[0, \pi / 2] \times[0,2 \pi]$, (where $\chi$ starting from $\xi_{3}=0$, see Fig. 3) then

$$
\left(\partial_{t}|\psi|^{2}\right)\left(0, \lambda \mathbf{e}_{2}\right) \leqslant-2 \int_{0}^{\pi / 4} d \beta \int_{0}^{2 \pi} d \chi b(\cos 2 \beta)(\sin 2 \beta)\left(1-\left|\psi_{3}(\lambda \cos \beta \sin \chi)\right|\right),
$$

because $\psi\left(\lambda \mathbf{e}_{2}\right)=1$ and $|\psi| \leqslant 1$. Choose $0<\beta_{1}<\beta_{2}<\pi / 4, \chi_{0} \in(0, \pi / 2)$ and $\lambda>0$ such that

$$
\lambda \cos \beta_{2} \sin \chi_{0}=a_{1}, \quad \lambda \cos \beta_{1}=a_{2}, \quad 2 \int_{\beta_{1}}^{\beta_{2}} b(\cos 2 \beta)(\sin 2 \beta) d \beta=c_{0}>0 .
$$

Then it follows from (2.5) that

$$
\left(\partial_{t}|\psi|^{2}\right)\left(0, \lambda \mathbf{e}_{2}\right) \leqslant-2 \int_{\beta_{1}}^{\beta_{2}} d \beta \int_{\chi_{0}}^{\pi-\chi_{0}} d \chi b(\cos 2 \beta)(\sin 2 \beta) \kappa=-\kappa c_{0}\left(\pi-2 \chi_{0}\right) .
$$

Since $\psi$ is symmetric around $\xi_{3}$ axis, we have

$$
\left(\partial_{t}|\psi|^{2}\right)(0, \xi) \leqslant-\kappa c_{0}\left(\pi-2 \chi_{0}\right), \quad \text { if } \xi \cdot \mathbf{e}_{3}=0 \text { and }|\xi|=\lambda .
$$

If we set $c_{1}=\kappa c_{0}\left(\pi-2 \chi_{0}\right)$, then there exist $\varepsilon>0, T>0$ and $\delta>0$ such that

$$
\left(\partial_{t}|\psi|^{2}\right)(t, \xi) \leqslant-c_{1} / 2, \quad \text { when }(t, \xi) \in[0, T] \times\left\{\xi \in \mathbb{R}^{3} ;|| \xi|-\lambda| \leqslant \delta,\left|\frac{\xi}{|\xi|} \cdot \mathbf{e}_{3}\right| \leqslant 2 \varepsilon\right\}:=[0, T] \times \Gamma
$$

because of the continuity of $\psi$ and $\partial_{t} \psi$ (see Theorem A.1 in Appendix A). 


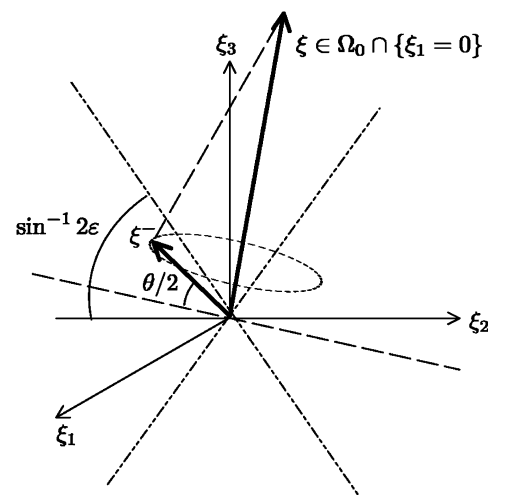

Fig. 4. $\xi \in \Omega_{0}$ and $\xi^{-}$almost orthogonal to $\xi_{3}$.

In what follows we use the notation $\xi^{-}=(\xi-|\xi| \sigma) / 2$ to obtain the microlocal time degenerate coercivity estimate. If $\left(t, \xi^{-}\right)$belongs to the region $[0, T] \times \Gamma$, then it follows from the mean value theorem that there exists a $\rho \in(0,1)$ such that

$$
1-\left|\psi\left(t, \xi^{-}\right)\right| \geqslant \frac{1-\left|\psi\left(t, \xi^{-}\right)\right|^{2}}{2}=\frac{1}{2}\left(1-\left|\psi\left(0, \xi^{-}\right)\right|^{2}-\left(\partial_{t}|\psi|^{2}\right)\left(\rho t, \xi^{-}\right) t\right) \geqslant \frac{c_{1}}{4} t .
$$

Set $R_{0}=(\lambda+\delta) / \varepsilon$ and

$$
\Omega_{0}=\left\{\xi \in \mathbb{R}^{3} ;|\xi| \geqslant R_{0},|1-| \frac{\xi}{|\xi|} \cdot \mathbf{e}_{3}|| \leqslant \frac{2 \varepsilon^{2}}{\pi^{2}}\right\} \quad \text { (see Fig. 4). }
$$

If $\sigma=(\theta, \phi)$, we notice that $\left|\xi^{-}\right|=|\xi| \sin (\theta / 2)$. Moreover, the fact that $\xi \in \Omega_{0}$ and $\sin \frac{\theta}{2} \leqslant(\lambda+\delta) /|\xi|$ implies $\left|\frac{\xi^{-}}{\mid \xi^{-1}} \cdot \mathbf{e}_{3}\right| \leqslant 2 \varepsilon$. Therefore, if $t \in[0, T]$ and if $h(\xi) \in L_{s}^{2}\left(\mathbb{R}^{3}\right)$, then we have the microlocal coercivity estimate in $\Omega_{0}$

$$
\begin{gathered}
\int_{\mathbb{R}^{3}}\left(\int_{\mathbb{S}^{2}} b\left(\frac{\xi}{|\xi|} \cdot \sigma\right)\left(1-\left|\psi\left(t, \xi^{-}\right)\right|\right) d \sigma\right)|h(\xi)|^{2} d \xi \gtrsim \int_{\Omega_{0}}\left(\int_{2 \sin ^{-1}(\lambda-\delta) /|\xi|}^{2 \sin ^{-1}(\lambda+\delta) /|\xi|} \theta^{-1-2 s} \frac{c_{1} t}{4} d \theta\right)|h(\xi)|^{2} d \xi \\
\gtrsim t \int_{\Omega_{0}}|\xi|^{2 s}|h(\xi)|^{2} d \xi .
\end{gathered}
$$

\subsubsection{The microlocal coercivity estimate in $\Omega_{0}^{c}$}

In this subsection, we consider the case when $\xi$ belongs to

$$
\Omega_{1}=\left\{\xi \in \mathbb{R}^{3} ;|1-| \frac{\xi}{|\xi|} \cdot \mathbf{e}_{3}||>\frac{2 \varepsilon^{2}}{\pi^{2}}\right\} \subset \Omega_{0}^{c} .
$$

Fix an arbitrary $\omega \in \mathbb{S}^{2} \cap \Omega_{1} \cap\left\{\omega \cdot \mathbf{e}_{3} \geqslant 0\right\}$. Take a $\lambda>0$ such that $\lambda \sin \gamma=\left(a_{1}+a_{2}\right) / 2$, where $\gamma>2 \varepsilon / \pi$ is the angle between $\omega$ and $\mathbf{e}_{3}$. If we take the polar coordinate $\sigma=(\theta, \phi) \in[0, \pi / 2] \times[0,2 \pi]$ with the pole $\omega=\xi /|\xi|$ and $\phi$ starting from the plane $\xi_{1}=0$ (see Fig. 5), then we have

$$
\xi^{-} \cdot \mathbf{e}_{3}=\left|\xi^{-}\right|\left(\cos \frac{\theta}{2} \cos \phi \sin \gamma+\sin \frac{\theta}{2} \cos \gamma\right),
$$

where $\xi^{-}=(\xi-|\xi| \sigma) / 2$. There exist $\delta=\delta_{\omega}>0, \phi_{\omega} \in(0, \pi / 4]$ and $\theta_{\omega} \in(0, \pi / 4]$ such that

$$
a_{1}<(\lambda-\delta) \cos \left(\theta_{\omega} / 2\right) \cos \phi_{\omega} \sin \gamma<(\lambda+\delta)\left(\sin \gamma+\tan \theta_{\omega} / 2\right)<a_{2} .
$$

Put $R_{\omega} \sin \theta_{\omega} / 2=\lambda+\delta_{\omega}$ and let $\xi=|\xi| \omega$ with $|\xi| \geqslant R_{\omega}$. If $\left|\xi^{-}\right|=|\xi| \sin \theta / 2 \in[\lambda-\delta, \lambda+\delta]$ and $|\xi| \geqslant R_{\omega}$, then $\theta \leqslant \theta_{\omega}$. Moreover, when $|\phi| \leqslant \phi_{\omega}$ we have 


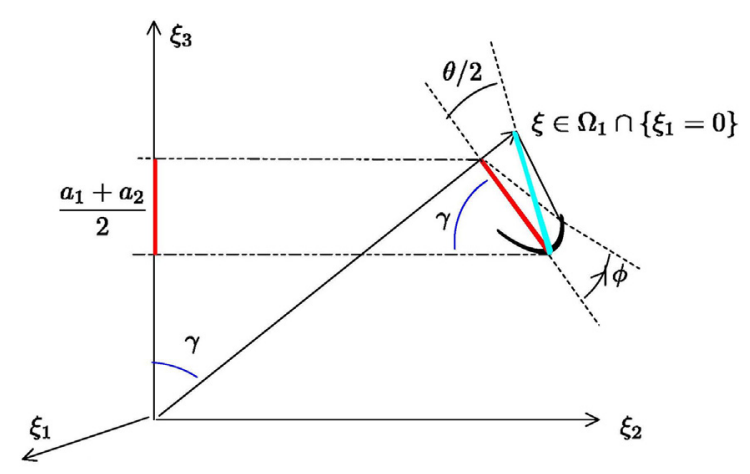

Fig. 5. Case: $\left|\xi^{-} \cdot \mathbf{e}_{3}\right| \cos (\theta / 2)=\left(a_{1}+a_{2}\right) / 2$ and $\xi^{-} \in\left\{\xi_{1}=0\right\}$.

$$
\xi^{-} \cdot \mathbf{e}_{3} \in\left(a_{1}, a_{2}\right)
$$

Since (2.8) still holds for other $\tilde{\gamma}$ close to $\gamma$, we have (2.9) for any $\xi$ belonging to a conic neighborhood of $\omega$

$$
\Gamma_{\omega}=\left\{\xi \in \mathbb{R}^{3} ;\left|\frac{\xi}{|\xi|}-\omega\right|<\varepsilon_{\omega},|\xi| \geqslant R_{\omega}\right\}
$$

with a sufficiently small $\varepsilon_{\omega}>0$, if $(\theta, \phi)$ varies in the same region as above. Since $\psi(t, \xi)$ is continuous, it follows from (2.5) that there exists a $T_{\omega}>0$ such that for any $t \in\left[0, T_{\omega}\right]$ we have

$$
\left|\psi\left(t, \xi^{-}\right)\right| \leqslant 1-\frac{\kappa}{2} \quad \text { if }\left|\xi^{-}\right| \in[\lambda-\delta, \lambda+\delta] \text { and } \xi^{-} \cdot \mathbf{e}_{3} \in\left[a_{1}, a_{2}\right] .
$$

Therefore

$$
\begin{aligned}
\int_{\mathbb{R}^{3}}\left(\int_{\mathbb{S}^{2}} b\left(\frac{\xi}{|\xi|} \cdot \sigma\right)\left(1-\mid \psi\left(t, \xi^{-} \mid\right)\right) d \sigma\right)|h(\xi)|^{2} d \xi & \gtrsim \int_{\Gamma_{\omega}}\left(\int_{-\phi_{\omega}}^{\phi_{\omega}} d \phi \int_{2 \sin ^{-1}(\lambda-\delta) /|\xi|}^{2 \sin ^{-1}(\lambda+\delta) /|\xi|} \theta^{-1-2 s} \frac{\kappa}{2} d \theta\right)|h(\xi)|^{2} d \xi \\
& \gtrsim \int_{\Gamma_{\omega}}|\xi|^{2 s}|h(\xi)|^{2} d \xi .
\end{aligned}
$$

The estimation for $\omega \in \mathbb{S}^{2} \cap \Omega_{1} \cap\left\{\omega \cdot \mathbf{e}_{3} \leqslant 0\right\}$ is similar, so that we omit it for brevity.

\subsubsection{The conclusion}

By means of the covering argument, we have for a sufficiently large $R>0$ and a sufficiently small $T>0$,

$$
\int_{\mathbb{R}^{3}}\left(\int_{\mathbb{S}^{2}} b\left(\frac{\xi}{|\xi|} \cdot \sigma\right)\left(1-\left|\psi\left(t, \xi^{-}\right)\right|\right) d \sigma\right)|h(\xi)|^{2} d \xi \gtrsim t \int_{\{|\xi| \geqslant R\}}|\xi|^{2 s}|h(\xi)|^{2} d \xi, \quad t \in[0, T] .
$$

This together with the coercivity estimate obtained in the first subsection concludes the proof of Lemma 1.4.

Before ending this subsection, we remark that if $\psi_{0}(\xi)=\int e^{-i v \cdot \xi} d F_{0}(v)$, then for a large $R>0$ we have the following degenerate coercivity estimate

$$
\int_{\mathbb{R}^{3}}\left(\int_{\mathbb{S}^{2}} b\left(\frac{\xi}{|\xi|} \cdot \sigma\right)\left(1-\left|\psi_{0}\left(\xi^{-}\right)\right|\right) d \sigma\right)|h(\xi)|^{2} d \xi \gtrsim \int_{\{|\xi| \geqslant R\}}\left(\left|\xi_{1}\right|^{2}+\left|\xi_{2}\right|^{2}+\left|\xi_{3}\right|\right)^{s}|h(\xi)|^{2} d \xi .
$$

Indeed, it follows from (2.7) that

$$
\xi^{-} \cdot \mathbf{e}_{3} \sim|\xi|\left(\theta \gamma \cos \phi+\theta^{2}\right) \sim|\xi|\left\{\theta\left(\frac{\left|\xi_{1}\right|^{2}+\left|\xi_{2}\right|^{2}}{|\xi|^{2}}\right)^{1 / 2} \cos \phi+\theta^{2}\right\}
$$

for sufficiently small $\gamma$ and $\theta$, and we have 


$$
\int_{\mathbb{S}^{2}} b\left(\frac{\xi}{|\xi|} \cdot \sigma\right)\left(1-\left|\psi_{0}\left(\xi^{-}\right)\right|\right) d \sigma \gtrsim \kappa \int_{\mathcal{A}} \theta^{-1-2 s} d \theta d \phi,
$$

where $\mathcal{A}=\left\{(\theta, \phi) ; \xi^{-} \cdot \mathbf{e}_{3} \in\left[a_{1}, a_{2}\right]\right\}$. However, this degenerate coercivity estimate is not sufficient to show the smoothing effect because the continuity in $\psi(t, \xi)$ does not imply $(2.10)$ with $\psi_{0}\left(\xi^{-}\right)$replaced by $\psi\left(t, \xi^{-}\right)$.

\section{Acknowledgement}

The authors would like to thank Cedric Villani for inspiring them to this topic.

\section{Appendix A}

In this appendix we first recall the result given in [6,14], and prove the continuity of $\partial_{t} \psi(t, \xi)$. For this, assume

$$
\exists \alpha_{0} \in(0,2] \quad \text { such that }(\sin \theta / 2)^{\alpha_{0}} b(\cos \theta) \sin \theta \in L^{1}((0, \pi / 2]),
$$

which is fulfilled for $b(\cos \theta)$ with (1.4) if $2 s<\alpha_{0}$. As stated in the proof of Theorem 1.3 in the introduction, it follows from the Bobylev formula that the Cauchy problem (1.1)-(1.2) is reduced to (1.10), if $\psi_{0}(\xi)=\int_{\mathbb{R}^{3}} e^{-i v \cdot \xi} d F_{0}(v)$ and $\psi(t, \xi)$ denotes the Fourier transform of the probability measure solution.

Theorem A.1. Assume that $b(\cos \theta)$ satisfies (A.1) for some $\alpha_{0} \in(0,2]$. Then for each $\alpha \in\left[\alpha_{0}, 2\right]$ and every $\psi_{0} \in \mathcal{K}^{\alpha}$ there exists a classical solution $\psi \in C\left([0, \infty), \mathcal{K}^{\alpha}\right)$ of the Cauchy problem (1.10). The solution is unique in the space $C\left([0, \infty), \mathcal{K}^{\alpha_{0}}\right)$. Furthermore, if $\alpha \in\left[\alpha_{0}, 2\right]$ and if $\psi(t, \xi), \varphi(t, \xi) \in C\left([0, \infty), \mathcal{K}^{\alpha}\right)$ are two solutions to the Cauchy problem (1.10) with initial data $\psi_{0}, \varphi_{0} \in \mathcal{K}^{\alpha}$, respectively, then for any $t>0$ we have

$$
\|\psi(t)-\varphi(t)\|_{\alpha} \leqslant e^{\lambda_{\alpha} t}\left\|\psi_{0}-\varphi_{0}\right\|_{\alpha}
$$

where

$$
\lambda_{\alpha}=2 \pi \int_{0}^{\pi / 2} b(\cos \theta)\left\{\cos ^{\alpha} \frac{\theta}{2}+\sin ^{\alpha} \frac{\theta}{2}-1\right\} \sin \theta d \theta .
$$

Furthermore, $\partial_{t} \psi(t, \xi)$ is continuous in $[0, \infty) \times \mathbb{R}^{3}$.

The assumption (A.1) with $\alpha=\alpha_{0}$ can be written as

$$
(1-\tau)^{\alpha_{0} / 2} b(\tau) \in L^{1}([0,1))
$$

by the change of variable $\tau=\cos \theta$. Theorem A.1 ameliorates Theorem 2.2 of [6], where (A.4) is assumed with $\alpha_{0} / 2$ replaced by $\alpha_{0} / 4$, see (2.6) of [6]. In what follows, we only prove the last statement of Theorem A.1 because other parts are already given in [14].

Proof of the continuity of $\partial_{t} \psi(t, \xi)$. If we put $\zeta=\left(\xi^{+} \cdot \frac{\xi}{|\xi|}\right) \frac{\xi}{\xi \xi \mid}$ and consider $\tilde{\xi}^{+}=\zeta-\left(\xi^{+}-\zeta\right)$ (which is symmetric to $\xi^{+}$on $\mathbb{S}^{2}$, see Fig. 6) as in [14], then the first equation of (1.10) can be written as

$$
\begin{aligned}
\partial_{t} \psi(t, \xi)= & \frac{1}{2} \int_{\mathbb{S}^{2}} b\left(\frac{\xi \cdot \sigma}{|\xi|}\right)\left(\psi\left(t, \xi^{+}\right)+\psi\left(t, \tilde{\xi}^{+}\right)-2 \psi(t, \zeta)\right) d \sigma \\
& +\int_{\mathbb{S}^{2}} b\left(\frac{\xi \cdot \sigma}{|\xi|}\right)(\psi(t, \zeta)-\psi(t, \xi)) d \sigma+\int_{\mathbb{S}^{2}} b\left(\frac{\xi \cdot \sigma}{|\xi|}\right) \psi\left(t, \xi^{+}\right)\left(\psi\left(t, \xi^{-}\right)-1\right) d \sigma \\
= & I_{1}(t, \xi)+I_{2}(t, \xi)+I_{3}(t, \xi) .
\end{aligned}
$$

Putting $\eta^{+}=\xi^{+}-\zeta$, we have, under the notation $d F_{t}(v)=f(t, v) d v$, 


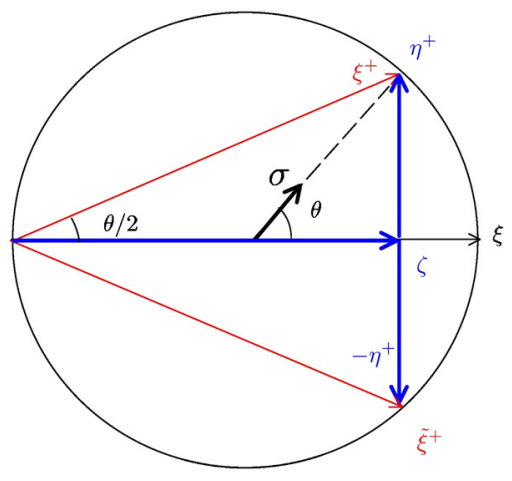

Fig. 6. $\cos \theta=\frac{\xi}{|\xi|} \cdot \sigma, \eta^{+}=\xi^{+}-\zeta$.

$$
\begin{aligned}
\left|\psi\left(t, \xi^{+}\right)+\psi\left(t, \tilde{\xi}^{+}\right)-2 \psi(t, \zeta)\right| & =\left|\int_{\mathbb{R}^{3}} e^{-i \zeta \cdot v}\left(e^{-i \eta^{+} \cdot v}+e^{i \eta^{+} \cdot v}-2\right) d F_{t}(v)\right| \\
& \leqslant \int_{\mathbb{R}^{3}}\left|e^{-i \zeta \cdot v}\right|\left(2-e^{-i \eta^{+} \cdot v}-e^{i \eta^{+} \cdot v}\right) d F_{t}(v) \\
& =2-\psi\left(t, \eta^{+}\right)-\psi\left(t,-\eta^{+}\right) \\
& \leqslant 2\|1-\psi(t)\|_{\alpha}\left|\eta^{+}\right|^{\alpha} \leqslant 2 e^{\lambda_{\alpha} t}\left\|1-\psi_{0}\right\|_{\alpha}(|\xi| \sin (\theta / 2))^{\alpha},
\end{aligned}
$$

because $\left|\eta^{+}\right|=\left|\xi^{+}\right| \sin (\theta / 2)$ and (A.2) with $\varphi_{0}=\varphi(t)=1$. Hence

$$
\left|I_{1}(t, \xi)\right| \leqslant 4 \pi e^{\lambda_{\alpha} t}\left\|1-\psi_{0}\right\|_{\alpha}|\xi|^{\alpha} \int_{0}^{\pi / 2} \sin ^{\alpha}(\theta / 2) b(\cos \theta) \sin \theta d \theta,
$$

which together with the Lebesgue convergence theorem shows

$$
\lim _{(t, \xi) \rightarrow\left(t_{0}, \xi_{0}\right)} I_{1}(t, \xi)=I_{1}\left(t_{0}, \xi_{0}\right) .
$$

In order to show similar estimates hold for $I_{2}, I_{3}$, we recall (19) of Lemma 2.1 in [14], that is, the fact that if $\varphi \in \mathcal{K}^{\alpha}$ then we have

$$
|\varphi(\xi)-\varphi(\xi+\eta)| \leqslant\|\varphi-1\|_{\alpha}\left(4|\xi|^{\alpha / 2}|\eta|^{\alpha / 2}+|\eta|^{\alpha}\right) \quad \text { for all } \xi, \eta \in \mathbb{R}^{3} .
$$

Thanks to this with $\eta=\zeta-\xi$,

$$
\left|I_{2}(t, \xi)\right| \leqslant 10 \pi e^{\lambda_{\alpha} t}\left\|1-\psi_{0}\right\|_{\alpha}|\xi|^{\alpha} \int_{0}^{\pi / 2} \sin ^{\alpha}(\theta / 2) b(\cos \theta) \sin \theta d \theta,
$$

because $|\zeta-\xi|=|\xi| \sin ^{2}(\theta / 2)$. Note that similar estimate holds for $I_{3}$. Hence, we obtain the continuity of $\partial_{t} \psi(t, \xi)$.

Proposition A.2. Assume that $b(\cos \theta)$ satisfies (A.1) for some $\alpha_{0} \in(0,2]$. If $F_{0} \in P_{2}\left(\mathbb{R}^{3}\right)$ then the unique measure solution $F_{t}(v) \in C\left([0, \infty), \tilde{P}_{2}\right)$ belongs to $P_{2}\left(\mathbb{R}^{3}\right)$ for each $t>0$, more precisely,

$$
\int|v|^{2} d F_{t}(v) \leqslant \int|v|^{2} d F_{0}(v) .
$$

Furthermore, if $\alpha_{0} \leqslant 1$ then the equality holds, that is, the energy is conserved. 
Proof. As a standard practice, we consider the increasing sequence of bounded collision kernels

$$
b_{n}(\cos \theta)=\min \{b(\cos \theta), n\}
$$

and denote by $\psi_{n}(t, \xi)$ the solution in $C\left([0, \infty) ; \mathcal{K}^{2}\right)$ to the Cauchy problem (1.10) with $b$ replaced by the cutoff $b_{n}$, for the same initial datum $\psi_{0}(\xi)=\int e^{-i v \cdot \xi} d F_{0}(v)$. It follows from Lemma 2.2 of [17] that

$$
\int|v|^{2} d F_{t}^{(n)}(v)=\int|v|^{2} d F_{0}(v)
$$

where $F_{t}^{(n)}=\mathcal{F}^{-1} \psi_{n}(t, \cdot)$. As proven in $[17,6,14]$, we have the equi-continuity of $\left\{\psi_{n}(t, \xi)\right\}$ on $[0, \infty) \times\{|\xi| \leqslant R\}$ for any fixed $R>0$. Since $\left|\psi_{n}\right| \leqslant 1$, the Ascoli-Arzelá theorem gives a convergent subsequence $\left\{\psi_{n_{k}}\right\}_{k=1}^{\infty}$ and the solution $\psi=\lim _{k \rightarrow \infty} \psi_{n_{k}}$. Take a $\chi(v)$ in $C_{0}^{\infty}\left(\mathbb{R}^{3}\right)$ satisfying $\chi=1$ on $\{|v| \leqslant 1\}$. Since $\psi_{n_{k}}(t) \rightarrow \psi(t)$ in $\mathcal{S}^{\prime}\left(\mathbb{R}^{3}\right)$ for each $t>0$, it follows from (A.9) that for any $m \in \mathbb{N}$

$$
\int|v|^{2} \chi\left(\frac{v}{m}\right) d F_{t}(v)=\lim _{k \rightarrow \infty} \int|v|^{2} \chi\left(\frac{v}{m}\right) d F_{t}^{\left(n_{k}\right)}(v) \leqslant \int|v|^{2} d F_{0}(v) .
$$

Letting $m \rightarrow \infty$ we obtain (A.7). In the mild singularity case, $\alpha_{0} \leqslant 1$, we can use Theorem 2 of [13] and its proof to show the reverse inequality of (A.7).

\section{References}

[1] R. Alexandre, L. Desvillettes, C. Villani, B. Wennberg, Entropy dissipation and long-range interactions, Arch. Ration. Mech. Anal. 152 (2000) $327-355$.

[2] R. Alexandre, M. Elsafadi, Littlewood Paley decomposition and regularity issues in Boltzmann homogeneous equations. I. Non cutoff and Maxwell cases, Math. Models Methods Appl. Sci. 15 (2005) 907-920.

[3] R. Alexandre, M. Elsafadi, Littlewood-Paley theory and regularity issues in Boltzmann homogeneous equations. II. Non cutoff case and non Maxwellian molecules, Discrete Contin. Dyn. Syst. 24 (2009) 1-11.

[4] R. Alexandre, Y. Morimoto, S. Ukai, C.-J. Xu, T. Yang, Smoothing effect of weak solutions for the spatially homogeneous Boltzmann equation without angular cutoff, Kyoto J. Math. 52 (2012) 433-463.

[5] L. Arkeryd, On the Boltzmann equation, Arch. Ration. Mech. Anal. 34 (1972) 1-34.

[6] M. Cannone, G. Karch, Infinite energy solutions to the homogeneous Boltzmann equation, Commun. Pure Appl. Math. 63 (2010) $747-778$.

[7] T. Carleman, Sur la théorie de l'équation intégrodifférentielle de Boltzmann, Acta Math. 60 (1933) 91-146.

[8] Y. Chen, L. He, Smoothing estimates for Boltzmann equation with full-range interactions: Spatially homogeneous case, Arch. Ration. Mech. Anal. 201 (2011) 501-548.

[9] L. Desvillettes, B. Wennberg, Smoothness of the solution of the spatially homogeneous Boltzmann equation without cutoff, Commun. Partial Differ. Equ. 29 (2004) 133-155.

[10] Z.H. Huo, Y. Morimoto, S. Ukai, T. Yang, Regularity of solutions for spatially homogeneous Boltzmann equation without Angular cutoff, Kinet. Relat. Models 1 (2008) 453-489.

[11] N. Jacob, Pseudo-Differential Operators and Markov Processes. Vol 1: Fourier Analysis and Semigroups, Imperial College Press, London, 2001.

[12] X. Lu, C. Mouhot, On measure solutions of the Boltzmann equation, part I: Moment production and stability estimates, J. Differ. Equ. 252 (2012) 3305-3363.

[13] X. Lu, B. Wennberg, Solutions with increasing energy for the spatially homogeneous Boltzmann equation, Nonlinear Anal., Real World Appl. 3 (2002) 243-258.

[14] Y. Morimoto, A remark on Cannone-Karch solutions to the homogeneous Boltzmann equation for Maxwellian molecules, Kinet. Relat. Models 5 (2012) 551-561.

[15] Y. Morimoto, S. Ukai, C.-J. Xu, T. Yang, Regularity of solutions to the spatially homogeneous Boltzmann equation without angular cutoff, Discrete Contin. Dyn. Syst., Ser. A 24 (2009) 187-212.

[16] Y. Morimoto, S. Ukai, Gevrey smoothing effect of solutions for spatially homogeneous nonlinear Boltzmann equation without angular cutoff, J. Pseudo-Differ. Oper. Appl. 1 (2010) 139-159.

[17] A. Pulvirenti, G. Toscani, The theory of the nonlinear Boltzmann equation for Maxwell molecules in Fourier representation, Ann. Mat. Pura Appl. 171 (1996) 181-204.

[18] H. Tanaka, Probabilistic treatment of the Boltzmann equation of Maxwellian molecules, Z. Wahrscheinlichkeitstheor. Verw. Geb. 46 (1978) 67-105.

[19] G. Toscani, C. Villani, Probability metrics and uniqueness of the solution to the Boltzmann equations for Maxwell gas, J. Stat. Phys. 94 (1999) 619-637.

[20] C. Villani, On a new class of weak solutions to the spatially homogeneous Boltzmann and Landau equations, Arch. Ration. Mech. Anal. 143 (1998) 273-307.

[21] C. Villani, A review of mathematical topics in collisional kinetic theory, in: S. Friedlander, D. Serre (Eds.), Handbook of Fluid Mathematical Fluid Dynamics, Elsevier Science, 2002.

[22] C. Villani, August 2008, Kyoto, private communication. 